LETTER TO THE EDITOR

\title{
First passage and arrival time densities for Lévy flights and the failure of the method of images
}

\author{
Aleksei V Chechkin $\dagger$, Ralf Metzler $\uparrow$, Vsevolod Y \\ Gonchar $\dagger$, Joseph Klafter \\ † Institute for Theoretical Physics NSC KIPT, Akademicheskaya st.1, 61108 \\ Kharkov, Ukraine \\ I NORDITA, Blegdamsvej 17, DK-2100 Copenhagen Ø, Denmark \\ $\ddagger$ School of Chemistry, Tel Aviv University, 69978 Tel Aviv Israel
}

\begin{abstract}
We discuss the first passage time problem in the semi-infinite interval, for homogeneous stochastic Markov processes with Lévy stable jump length distributions $\lambda(x) \sim \ell^{\alpha} /|x|^{1+\alpha}(|x| \gg \ell)$, namely, Lévy flights (LFs). In particular, we demonstrate that the method of images leads to a result, which violates a theorem due to Sparre Andersen, according to which an arbitrary continuous and symmetric jump length distribution produces a first passage time density (FPTD) governed by the universal long-time decay $\sim t^{-3 / 2}$. Conversely, we show that for LFs the direct definition known from Gaussian processes in fact defines the probability density of first arrival, which for LFs differs from the FPTD. Our findings are corroborated by numerical results.
\end{abstract}

Submitted to: J. Phys. A: Math. Gen.

PACS numbers: 05.40.Fb, 02.50.Ey, 05.60.Cd, 05.10.Gg

Lévy flights (LFs) and Lévy walks (LWs) are the prime example in the investigation of non-standard transport processes whose stationary solution does not converge towards the Boltzmann form [1, 2, 4, 3. Being subject to the generalised central limit theorem [5, 6], LFs correspond to a Markov process in which extremely long excursions can occur with appreciable probability, whereas in LWs long excursions are penalised through a time cost introduced via a spatiotemporal coupling [7] [8]. Applications of LFs and LWs range from the famed flight of an albatross [9], the spreading of spider-monkeys [10], or the grazing patterns of bacteria [11, over economical data 12 to molecular collisions 13 and plasmas [14. Despite their broad usage, the detailed behaviour of even the simpler, uncoupled LF processes, on which we concentrate in the following, in external potentials and under nontrivial boundary conditions is still not fully explored. Thus, there have recently been discovered bifurcations between multimodal states of the probability density function (PDF) of LFs in steeper than harmonic external fields, in whose presence also the variance becomes finite [15, 16], and rich band structures have been reported for LFs in periodic potentials 17 .

Of particular interest in random processes is the first passage time density (FPTD) [18, 19, 20, 21]. For LFs, the FPTD was determined through the method of images 
on a finite domain in reference [22], and with similar methods in reference [23]. These methods lead to results for the FPTD in the semi-infinite domain, whose long-time behaviour explicitly depends on the Lévy index $\alpha$. In contrast, a theorem due to Sparre Andersen proves that for any discrete-time random walk process starting at $x_{0} \neq 0$ with each step chosen from a continuous, symmetric but otherwise arbitrary distribution, the FPTD asymptotically decays as $\sim n^{-3 / 2}$ with the number $n$ of steps [21, 24, 25], being fully independent of the index of the LF, i.e., universal. In the case of a Markov process, the continuous time analogue of the Sparre Andersen result reads

$$
p(t) \sim t^{-3 / 2} .
$$

The analogous universality was proved by Frisch and Frisch for the special case in which an absorbing boundary is placed at the location of the starting point of the $\mathrm{LF}$ at $t>0$ [26], and numerically corroborated by Klafter and Zumofen [27. In the following, we demonstrate that the method of images is generally inconsistent with the universality of the FPTD, and therefore cannot be applied to solve FPTD-problems for LFs. We also show that for LFs the FPTD differs from the PDF for first arrival.

Let us start by recalling that an unbiased LF can be defined through the spacefractional diffusion equation for the PDF $W(x, t)[2,28,29$,

$$
\frac{\partial}{\partial t} W=D \frac{\partial^{\alpha}}{\partial|x|^{\alpha}} W(x, t) \therefore \quad \int_{-\infty}^{\infty} e^{\mathrm{i} k x} \frac{\partial^{\alpha} W}{\partial|x|^{\alpha}} \mathrm{d} x \equiv-|k|^{\alpha} W(k, t),
$$

where we define the fractional derivative $\partial^{\alpha} / \partial|x|^{\alpha}$ by its Fourier transform. (Here and in the following, we restrict ourselves to $1<\alpha<2$.) In position space, the fractional derivative is defined in terms of the convolution (see [15] for the case $\alpha=1$ )

$$
\frac{\partial^{\alpha}}{\partial|x|^{\alpha}} W(x, t) \equiv \frac{D}{\kappa} \frac{\partial^{2}}{\partial x^{2}} \int_{-\infty}^{\infty} \frac{W\left(x^{\prime}, t\right)}{\left|x-x^{\prime}\right|^{\alpha-1}} \therefore \kappa \equiv 2 \Gamma(2-\alpha)\left|\cos \frac{\pi \alpha}{2}\right| \cdot(3)
$$

Equivalently, LFs can be described in terms of continuous time random walks with long-tailed jump length distributions $\lambda(x) \sim \ell^{\alpha} /|x|^{1+\alpha}$ [7] 30. The associated PDF $W(x, t)$ for natural boundary conditions $\left(\lim _{|x| \rightarrow \infty} W(x, t)=0\right)$ with initial condition $\delta(x)$ is the Lévy stable law $W(x, t)=\int_{-\infty}^{\infty} \exp \left(-\mathrm{i} k x-D|k|^{\alpha} t\right) \mathrm{d} k /(2 \pi)$ [5] [6]. In Fourier-Laplace space 31, this PDF corresponds to $W(k, s)=\left(s+D|k|^{\alpha}\right)^{-1}$. A characteristic of LFs is the divergence of the variance of both $W(x, t)$ and $\lambda(x)$. Equipping equation (2) with a $\delta$-sink of strength $p_{\mathrm{fa}}(t)$, we obtain the diffusion-reaction equation for the non-normalised density function $f(x, t)$,

$$
\frac{\partial}{\partial t} f(x, t)=D \frac{\partial^{\alpha}}{\partial|x|^{\alpha}} f(x, t)-p_{\mathrm{fa}}(t) \delta(x),
$$

from which by integration over the unrestricted space, we find the quantity

$$
p_{\mathrm{fa}}(t)=-\frac{\mathrm{d}}{\mathrm{d} t} \int_{-\infty}^{\infty} f(x, t) \mathrm{d} x,
$$

i.e., $p_{\mathrm{fa}}(t)$ is the negative time derivative of the survival probability. By definition of the sink term, $p_{\mathrm{fa}}(t)$ is the PDF of first arrival: once a random walker arrives at the sink, it is annihilated. By solving equation (4) through standard methods (determining the homogeneous and inhomogeneous solutions), it is straightforward to calculate the solution $f$ in terms of the propagator $W$ of equation (21) with initial condition $f(x, 0)=\delta\left(x-x_{0}\right)$ yielding $f(k, u)=\left[e^{\mathrm{i} k x_{0}}+p_{\mathrm{fa}}(u)\right] /\left(s+D|k|^{\alpha}\right)$, from 
which, in turn, we find that $p_{\mathrm{fa}}(t)$ satisfies the chain rule $\left(p_{\mathrm{fa}}\right.$ implicitly depending on $\left.x_{0}\right)$

$$
W\left(-x_{0}, t\right)=\int_{0}^{t} p_{\mathrm{fa}}(\tau) W(0, t-\tau) \mathrm{d} \tau
$$

which corresponds to the Laplace space relation $p_{\mathrm{fa}}(s)=W\left(-x_{0}, s\right) / W(0, s)$. Equation (6) is well-known and for any sufficiently well-behaved continuum diffusion process is commonly employed to define the FPTD [19, 21.

For Gaussian processes with propagator $W(x, t)=1 / \sqrt{4 \pi D t} \exp \left(-x^{2} /[4 D t]\right)$, one obtains by direct integration of the diffusion equation with appropriate boundary condition the FPTD 21]

$$
p(t)=x_{0}\left(4 \pi D t^{3}\right)^{-1 / 2} \exp \left(-x_{0}^{2} /(4 D t)\right),
$$

including the asymptotic behaviour $p(t) \sim t^{-3 / 2}$ for $t \gg x_{0}^{2} /(4 D)$. In this Gaussian case, the quantity $p_{\mathrm{fa}}(t)$ is equivalent to the FPTD. From a random walk perspective, this is due to the fact that individual steps are of the same increment, and the jump length statistics therefore ensures that the walker cannot hop across the sink in a long jump without actually hitting the sink and being absorbed. This behaviour becomes drastically different for Lévy jump length statistics: There, the particle can easily cross the sink in a long jump. Thus, before eventually being absorbed, it can pass by the sink location numerous times, and therefore the statistics of the first arrival will be different from the one of the first passage. In fact, with $W(x, s)=(2 \pi)^{-1} \int_{-\infty}^{\infty} e^{\mathrm{i} k x}\left(s+D|k|^{\alpha}\right)^{-1} \mathrm{~d} k$, we find

$$
p_{\mathrm{fa}}(s)=1-\frac{\int_{0}^{\infty}\left(1-\cos k x_{0}\right) /\left(s+D k^{\alpha}\right) \mathrm{d} k}{\int_{0}^{\infty} 1 /\left(s+D k^{\alpha}\right) \mathrm{d} k}
$$

by use of the de Moivre identity $\exp (\mathrm{i} z)=\cos z+\mathrm{i} \sin z$. With $\int_{0}^{\infty}\left(s+D k^{\alpha}\right)^{-1} \mathrm{~d} k=$ $\pi s^{1 / \alpha-1} /\left(\alpha D^{1 / \alpha} \sin (\pi / \alpha)\right)$ and

$$
\int_{0}^{\infty} \frac{1-\cos k x_{0}}{s+D k^{\alpha}} \sim \frac{\Gamma\left((2-\alpha) \sin (\pi(2-\alpha) / 2) x_{0}^{\alpha-1}\right.}{(\alpha-1) D}, \quad s \rightarrow 0, \alpha>1,
$$

we obtain the limiting form

$$
p_{\mathrm{fa}}(s) \sim 1-x_{0}^{\alpha-1} s^{1-1 / \alpha} D^{-1+1 / \alpha} \tilde{\Lambda}(\alpha),
$$

where $\tilde{\Lambda}(\alpha)=\alpha \Gamma(2-\alpha) \sin (\pi(2-\alpha) / 2) \sin (\pi / \alpha) /(\alpha-1)$. We note that the same result is obtained by the exact expressions for $W\left(x_{0}, s\right)$ and $W(0, s)$ in terms of Fox $H$-functions and systematic expansion 32. The inverse Laplace transform of the small $s$-behaviour (9) can be obtained by completing (9) to an exponential, and then computing the Laplace inversion by the identification $e^{z}=H_{0,1}^{1,0}[z \mid(0,1)]$ with the Fox $H$-function [32], for which the exact Laplace inversion can be performed 33. Finally, a series expansion of this result leads to the long- $t$ form

$$
p_{\mathrm{fa}}(t) \sim C(\alpha) \frac{x_{0}^{\alpha-1}}{D^{1-1 / \alpha} t^{2-1 / \alpha}}
$$

with $C(\alpha)=\alpha \Gamma(2-\alpha) \Gamma(2-1 / \alpha) \sin (\pi[2-\alpha] / 2) \sin ^{2}(\pi / \alpha) /\left(\pi^{2}(\alpha-1)\right)$. Clearly, in the Gaussian limit, the required asymptotic form $p(t) \sim x_{0} / \sqrt{4 \pi D t^{3}}$ for the FPTD is consistently recovered, whereas in the general case the result (10) is slower than in the universal FPTD behaviour in equation (11), as it should as the $\delta$-trap used in equation (44) to define the first arrival for LFs is weaker than the absorbing wall used 


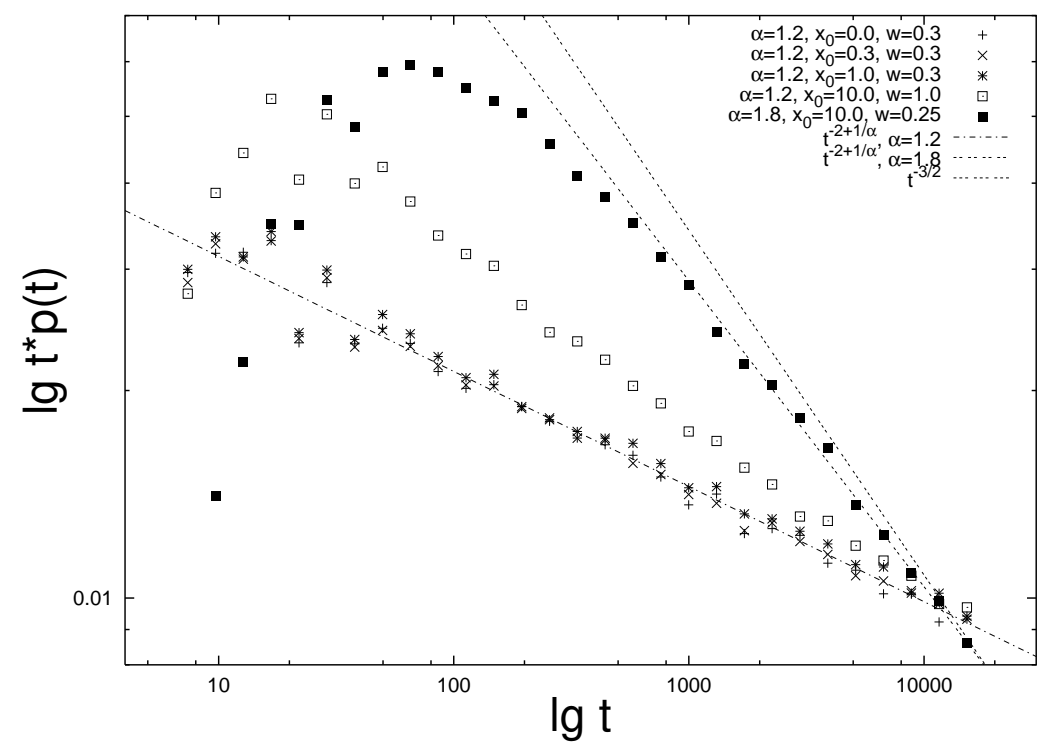

Figure 1. First arrival PDF for $\alpha=1.2$ demonstrating the $t^{-2+1 / \alpha}$ scaling, for optimal trap width $w=0.3$. For comparison, we show the same scaling for $\alpha=1.8$, and the power-law $t^{-3 / 2}$ corresponding to the FPTD. The behaviour for too large $w=1.0$ shows a shift of the decay towards the $-3 / 2$ slope. Note that on the abscissa we plot $\lg t p(t)$. Note also that for the initial condition $x_{0}=0.0$, the trap becomes activated after the first step, consistent with [27.

to properly define the FPTD. For LFs, the PDF for first arrival thus scales like (10) (i.e., it explicitly depends on the index $\alpha$ of the underlying Lévy process), and, as shown below, it differs from the corresponding FPTD.

Before calculating this FPTD, we first demonstrate the validity of equation (10) by means of a simulation the results of which are shown in figure 1 Random jumps with LF jump length statistics are performed, and a particle is removed when it hits a certain interval of width $w$ around the sink; for our simulations we found an optimum value $w \approx 0.3$. As seen in figure 1 (note that we plot $\lg t p(t) !)$ and for analogous results not shown here, relation (10) is nicely fulfilled for all $1<\alpha<2$, whereas for larger $w$, the slope increases.

The proper dynamical formulation of an LF on the semi-infinite interval with an absorbing boundary condition at $x=0$, and thereby the determination of the FPTD, has to make sure that in terms of above random walk picture jumps across the sink are forbidden. This can be consistently achieved by setting $f(x, t) \equiv 0$ on the left semi-axis, i.e., actually removing the particle when it crosses the point $x=0$. This formally corresponds to the modified dynamical equation

$$
\frac{\partial f(x, t)}{\partial t}=\frac{D}{\kappa} \frac{\partial^{2}}{\partial x^{2}} \int_{0}^{\infty} \frac{f\left(x^{\prime}, t\right)}{\left|x-x^{\prime}\right|^{\alpha-1}} \mathrm{~d} x^{\prime} \equiv \frac{\partial^{2}}{\partial x^{2}} \mathcal{F}(x, t)
$$

in which the fractional integral is truncated to the semi-infinite interval. After Laplace transformation and integrating over $x$ twice, one obtains

$$
\int_{0}^{\infty} K\left(x-x^{\prime}, s\right) f\left(x^{\prime}, s\right) d x^{\prime}=\left(x-x_{0}\right) \Theta\left(x-x_{0}\right)-x p(s)-\mathcal{F}(0, s),
$$

where $p(t)$ is the FPTD and the kernel $K(x, s)=s x \Theta(x)-\left(\kappa|x|^{\alpha-1}\right)$. This equation is formally of the Wiener-Hopf type of the first kind 34]. After some manipulations 


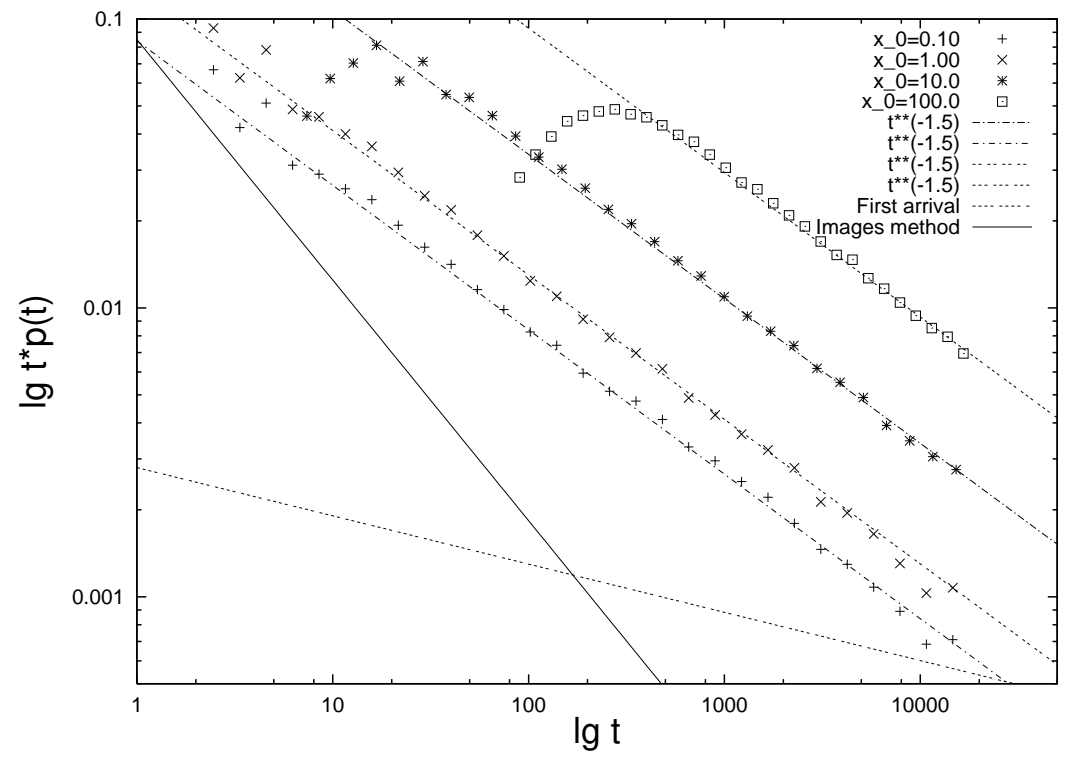

Figure 2. Numerical results for the FPTD process on the semi-infinite domain, for an LF with Lévy index $\alpha=1.2$. Note that on the abscissa, we plot $t p(t)$. For all initial conditions $x_{0}=0.101 .00,10.0$, and 100.0 the universal slope $-3 / 2$ in the $\log _{10}-\log _{10}$ plot is nicely reproduced, and it is significantly apart from the two slopes predicted by the images method and the direct definition of the FPTD.

similar to those applied in reference [27, we arrive at the asymptotic expression $p(s) \simeq 1-C s^{1 / 2}$, where $C=$ const, in accordance with the universal behaviour (II) and with the findings in reference [27. Thus, the dynamic equation (11) consistently phrases the FPTD problem for LFs. We note that due to the truncation of the fractional integral it was not possible to modify the well-established GrünwaldLetnikov scheme [35] to numerically solve equation (11) with enough computational efficiency to numercially obtain the direct solution for $f(x, t)$. However, to corroborate the validity of the Sparre Anderson-universality, we perform a simulation of an LF in the presence of an absorbing wall, i.e., random jumps with LF jump length statistics are performed along the right semi-axis, and a particle is removed when it jumps across the origin to the left semi-axis. Results of such a detailed random walk study are displayed in figures 2 and 3 The expected universal $t^{-3 / 2}$ scaling is nicely obtained for various initial conditions and $\alpha$. Clearly, the scaling for the first arrival as well as the image method-FPTD derived below are significantly different.

We now demonstrate that the method of images produces a result, which is neither consistent with the universal behaviour of the FPTD (11) nor with the behaviour of the PDF of first arrival (10), Given the initial condition $\delta\left(x-x_{0}\right)$, the solution $f_{\mathrm{im}}(x, t)$ for the absorbing boundary value problem with the analogous Dirichlet condition $f_{\mathrm{im}}(0, t)=0$ according to the method of images is given via the difference [20, 21]

$$
f_{\mathrm{im}}(x, t)=W\left(x-x_{0}, t\right)-W\left(x+x_{0}, t\right),
$$

in terms of the free propagator $W$, i.e., a negative image solution originating at $-x_{0}$ balances the probability flux across the absorbing boundary. The corresponding pseudo-FPTD is then calculated in the same way as in equation (5). For the image solution in Fourier-Laplace space, we obtain

$$
f_{\mathrm{im}}(k, s)=\left[2 \mathrm{i} \sin \left(k x_{0}\right)\right] /\left(s+D|k|^{\alpha}\right),
$$




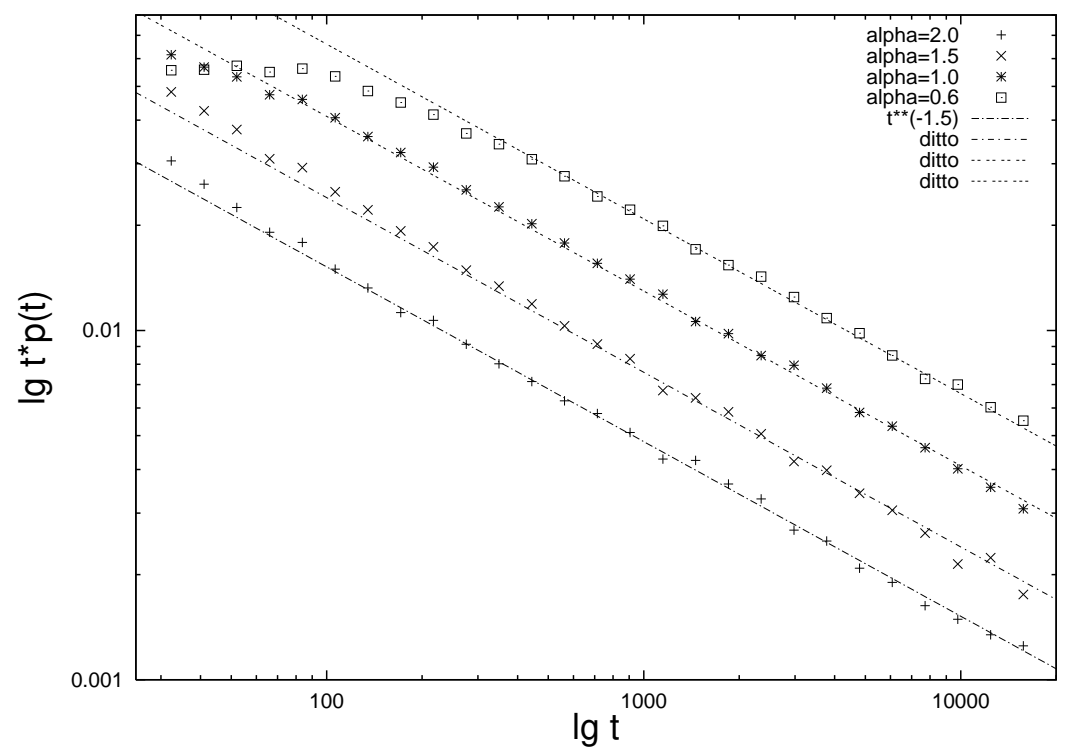

Figure 3. Same as in figure 2 for $\alpha=2.0,1.5,1.0$, and 0.6 , and for the initial condition $x_{0}=10.0$. Again, the universal $\sim t^{-3 / 2}$ behaviour is obtained.

for a process which starts at $x_{0}>0$ and takes place in the right half space. In Laplace space, the image method-FPTD becomes

$$
p_{\text {im }}(s)=1-s \int_{0}^{\infty} \mathrm{d} x \int_{-\infty}^{\infty} \frac{\mathrm{d} k}{2 \pi} e^{-\mathrm{i} k x} \frac{2 \mathrm{i} \sin k x_{0}}{s+D|k|^{\alpha}} .
$$

After some transformations, we arrive at

$$
p_{\text {im }}(s)=1-2 / \pi \int_{0}^{\infty} \mathrm{d} \xi \sin \left(\xi s^{1 / \alpha} x_{0} / D^{1 / \alpha}\right) /\left[\xi\left(1+\xi^{\alpha}\right)\right] .
$$

In the limit of small $s$, this expression reduces to $p_{\text {im }}(s) \sim 1-\Lambda(\alpha) x_{0} D^{-1 / \alpha} s^{1 / \alpha}$, with $\Lambda(\alpha)=(2 / \pi) \int_{0}^{\infty}\left(1+\xi^{\alpha}\right)^{-1} \mathrm{~d} \xi=2 /(\alpha \sin (\pi / \alpha))$. Following the same procedure as outlined above, we find the long- $t$ form

$$
p_{\text {im }}(t) \sim 2 \Gamma(1 / \alpha) x_{0} /\left(\pi \alpha D^{1 / \alpha} t^{1+1 / \alpha}\right)
$$

for the image method-FPTD. In the Gaussian limit $\alpha=2$, expression (17) produces $p_{\mathrm{im}}(t) \sim x_{0} / \sqrt{4 \pi D t^{3}}$, in accordance to equation (7). Conversely, for general $1<\alpha<2$, $p_{\text {im }}(t)$ according to equation (17) would decay faster than $\sim t^{-3 / 2}$. The method of images breaks down for LFs due to their special non-local nature, displayed by the integrals in equations (2) and (3), and (11), namely having a long-tailed jump length distribution. This leads to leapovers beyond the absorbing boundary. The method of images is expected to work when the boundary is also a turning poing of the trajectory, as actually happens for nearest neighbours random walks, or the Wiener process.

Qualitatively, the following argument may be brought forth in favour of the observed universality of the LF-FPTD: the long-time decay is expected to be governed by short-distance jump events, corresponding to the central region of very small jump lengths for the Lévy stable jump length distribution. But this region is, apart from a prefactor, indistinguishable from the Gaussian distribution, and therefore the longtime behaviour should in fact be the same for any continuous jump length distribution 
$\lambda(x)$. In fact, the universal law (11) can only be modified in the presence of nonMarkov effects such as broad waiting time processes or spatiotemporally coupled walks [2, 21, 7, 36, 37. In terms of the special case covered by the theorem of Frisch and Frisch [26], in which the absorbing boundary coincides with the initial condition, we can understand the general situation for finite $x_{0}>0$, as in the long-time limit, the distance $x_{0}$ becomes negligible in comparison to the diffusion length $\langle|x(t)|\rangle \sim t^{1 / \alpha}$ : therefore the asymptotic behaviour is necessarily governed by the same universality.

Concluding, we demonstrated that the method of images, which has been developed a powerful tool in Gaussian diffusion also beyond the homogeneous case [20, 21] and in the presence of long-tailed waiting times [2, 36, 37, fails for LF processes, leading to a false result for the FPTD. Moreover, we showed that for such broad jump length statistics, the PDF of first arrival at a point differs from the FPTD. We also provided a framework in terms of a truncated fractional diffusion equation to solve the FPTD problem for an LF. This study is expected to significantly contribute to the understanding of the, at instances, non-trivial behaviour of LFs.

\section{Acknowledgments}

We are happy to acknowledge discussions with Igor Sokolov. We also acknowledge financial support through the INTAS project 00-0847 from the European Commission.

\section{References}

[1] Bouchaud J-P and Georges A 1990 Phys. Rep. 195127

[2] Metzler R and Klafter J 2000 Phys. Rep. 339, 1

[3] Klafter J, Shlesinger M F and Zumofen G 1996 Phys. Today 49(2), 33

[4] Shlesinger M F, Zaslavsky G M and Frisch U (eds) 1995 Lévy flights and related topics in physics Lecture Notes in Physics 450 (Springer-Verlag/Berlin)

[5] Lévy P 1954 Théorie de l'addition des variables aléatoires (Gauthier-Villars/Paris)

[6] Gnedenko BV and Kolmogorov AN 1954 Limit Distributions for Sums of Random Variables (Addison-Wesley/Reading, MA)

[7] Klafter J, Blumen A and Shlesinger MF 1987 Phys. Rev. A 35, 3081

[8] Sokolov IM and Metzler R 2003, Phys. Rev. E 67, 010101(R)

[9] Viswanathan GM, Afanasyev V, Buldyrev SV, Murphey EJ, Prince PA and Stanley HE 1996 Nature 381413

[10] G. Ramos-Fernandez, J. L. Mateos, O. Miramontes, G. Cocho, H. Larralde, and B. Ayala-Orozco, preprint cond-mat/0301019

[11] Levandowsky M, White BS and Schuster FL 1997 Acta Protozool. 36, 237

[12] Mandelbrot BB 1963, J. Bus. 36, 394; 1966 ibid. 39, 242; 1967 ibid. 40, 393

[13] Carati A, Galgani L and Pozzi B 2003 Phys. Rev. Lett. 90, 010601

[14] Chechkin AV, Gonchar VY and Szydlowsky M 2002 Phys. Plasma 9, 78

[15] Chechkin AV, Gonchar V, Klafter J, Metzler R and Tanatarov L 2002 Chem. Phys. 284233

[16] Chechkin AV, Gonchar V, Klafter J, Metzler R and Tanatarov L 2003 Phys. Rev. E $67010102(\mathrm{R})$

[17] Brockmann, D and Geisel T, 2003 Phys. Rev. Lett. 90170601

[18] Schrödinger, E 1915 Physikal. Zeitschrift 16289

[19] Hughes BD 1995 Random Walks and Random Environments, Volume 1: Random Walks (Oxford University Press/Oxford)

[20] Feller W 1968 An Introduction to Probability Theory and Its Applications (John Wiley \& Sons/New York)

[21] Redner S 2001 A guide to first-passage processes (Cambridge University Press/Cambridge (UK))

[22] Montroll EW and West BJ 1976, in Fluctuation phenomena, edited by Montroll EW and Lebowitz JL (North-Holland, Amsterdam)

[23] Gitterman M 2000 Phys. Rev. E 62, 6065

[24] Sparre Andersen E 1953 Math. Scand. 1, 263

[25] Sparre Andersen E 1954 Math. Scand. 2, 195 
[26] Frisch U and Frisch H 1995, in 4

[27] Zumofen G and Klafter J 1995 Phys. Rev. E 512805

[28] Fogedby HC 1994 Phys. Rev. Lett. 732517

[29] Jespersen S, Metzler R and Fogedby HC 1999 Phys. Rev. E 592736

[30] Fogedby HC 1994 Phys. Rev. E 50, 1657

[31] We denote the Laplace and Fourier transforms through the explicit use of the image variables: $p(s) \equiv \int_{0}^{\infty} p(t) e^{-s t} \mathrm{~d} t$, and $f(k, t) \equiv \int_{-\infty}^{\infty} f(x, t) e^{\mathrm{i} k x} \mathrm{~d} x$.

[32] Mathai AM and Saxena RK 1978 The H-function with Applications in Statistics and Other Disciplines (Wiley Eastern Ltd./New Delhi)

[33] Glöckle WG and Nonnenmacher TF 1993 J. Stat. Phys. 71755

[34] Gakhov FD, 1966, Boundary value problems (Pergamon Press, Oxford)

[35] Gorenflo R, Mainardi F, Moretti D, Pagnini G and Paradisi P 2002, Chem. Phys. 284, 521

[36] Scher H, Margolin G, Metzler R, Klafter J and Berkowitz B 2002, Geophys. Res. Lett. 29, 10.1029/2001GL014123

[37] Metzler R and Klafter J 2003, Biophys. J. 85(4) at press 